\title{
Oversizing and Restenosis with Self-Expanding Stents in Iliofemoral Arteries
}

\author{
Ardan M. Saguner • Tobias Traupe $\cdot$ Lorenz Räber • \\ Nina Hess · Yara Banz • Arhan R. Saguner • \\ Nicolas Diehm $\cdot$ Otto M. Hess
}

Received: 6 June 2011/ Accepted: 4 September 2011/Published online: 18 October 2011

(C) Springer Science+Business Media, LLC and the Cardiovascular and Interventional Radiological Society of Europe (CIRSE) 2011

\begin{abstract}
Purpose Uncoated self-expanding nitinol stents (NS) are commonly oversized in peripheral arteries. In current practice, 1-mm oversizing is recommended. Yet, oversizing of NS may be associated with increased restenosis. To provide further evidence, NS were implanted in porcine iliofemoral arteries with a stent-to-artery-ratio between 1.0 and 2.3. Besides conventional uncoated NS, a novel selfexpanding NS with an antiproliferative titanium-nitrideoxide (TiNOX) coating was tested for safety and efficacy. Methods Ten uncoated NS and six TiNOX-coated NS (5-6 mm) were implanted randomly in the iliofemoral artery of six mini-pigs. After implantation, quantitative angiography (QA) was performed for calculation of artery and minimal luminal diameter. Follow-up was performed by QA and histomorphometry after 5 months.

Results Stent migration, stent fracture, or thrombus formation were not observed. All stents were patent at followup. Based on the location of the stent (iliac/femoral) and the stent-to-artery-ratio, stent segments were divided into "normal-sized" (stent-to-artery-ratio $<1.4, \mathrm{n}=12$ ) and
\end{abstract}

Otto M. Hess-deceased.

A. M. Saguner · T. Traupe $\cdot$ L. Räber $\cdot$ N. Hess ·

A. R. Saguner · N. Diehm · O. M. Hess

Swiss Cardiovascular Center, University Hospital,

Bern, Switzerland

\section{A. M. Saguner $(\bowtie)$}

Clinic for Cardiology, University Hospital, Raemistrasse 100, 8091 Zurich, Switzerland

e-mail: ardan.saguner@usz.ch

Y. Banz

Institute of Pathology, University of Bern, Bern, Switzerland "oversized" (stent-to-artery-ratio $\geq 1.4, \mathrm{n}=9$ ). All stent segments expanded to their near nominal diameter during follow-up. Normal-sized stent segments increased their diameter by $6 \%$ and oversized segments by $29 \%$. A significant correlation between oversizing and restenosis by both angiography and histomorphometry was observed. Restenosis rates were similar for uncoated NS and TiNOXcoated NS.

Conclusions TiNOX-coated NS are as safe and effective as uncoated NS in the porcine iliofemoral artery. All stents further expand to near their nominal diameter during follow-up. Oversizing is linearly and positively correlated with neointimal proliferation and restenosis, which may not be reduced by TiNOX-coating.

Keywords Catheterization, peripheral $\cdot$ Stent $\cdot$ Nitinol · Titanium-nitride-oxide $\cdot$ Porcine

\section{Introduction}

Restenosis is the main drawback of endovascular treatment of the iliac and femoral arteries [1-4]. Conflicting experimental and clinical results have been reported for patency rates and restenosis in the iliofemoral arteries with different stent designs [5-8]. Currently, self-expanding nitinol stents are used for the treatment of peripheral artery disease. Selfexpanding stents are commonly oversized to ensure optimal wall apposition and to prevent stent migration $[8,9]$. In practice, 1-mm oversizing is usually recommended. However, stent oversizing, determined as the ratio of nominal stent diameter to vessel diameter at implantation, may cause significant neointimal proliferation and restenosis mainly in the superficial femoral arteries, but also in iliac arteries as reported in a recent porcine study [8]. 
Titanium-nitride-oxide-coated stents (TiNOX) are biologically inert compared with the nickel component of nitinol stents and can significantly reduce neointimal hyperplasia in coated stainless steel stents [10]. Data about self-expanding TiNOX-coated stents in the iliofemoral arterial bed are scarce. Thus, the purpose of the present study was to test safety and efficacy of self-expanding TiNOX-coated nitinol stents in direct comparison to selfexpanding uncoated nitinol stents in porcine iliofemoral arteries, investigating the impact of stent oversizing on restenosis and long-term outcome.

\section{Materials and Methods}

This prospective, controlled animal study was approved by the local animal ethical committee. It conforms to the guidelines established in the "Position of the American Heart Association on Research Animal Use" adopted by the American Heart Association on November 11, 1984. We used self-expanding uncoated peripheral nitinol stents $(\mathrm{n}=10)$ and self-expanding TiNOX-coated peripheral nitinol stents $(n=6)$.

TiNOX stents feature a flexible helicoidal stainless steel shaft with open cell design and a smooth electropolish finish. The thin coating consists of a titanium-nitride-oxide alloy. It functions as a barrier minimizing the leakage of metal residues, mostly nickel, into the arterial wall and attenuating electrical conductivity. This feature potentially minimizes cytotoxicity and provides beneficial effects on hemocompatibility. The stent is enclosed within a sheath before deployment at the target lesion. Implanted stents had the following sizes, as provided by the manufacturers (Biotronik AG, Bülach, Switzerland; Hexacath SA, Paris, France): $5 \times 30-\mathrm{mm}, 6 \times 40-\mathrm{mm}$, and $6 \times 60-\mathrm{mm}$. They were implanted in six mini-pigs of either sex (body weight $45 \mathrm{~kg}$ ), depending on the size and anatomy of the iliofemoral arterial bed. Stent implantation was randomized to prevent implantation bias. Stent oversizing was determined as the ratio of nominal stent diameter to artery diameter at implantation (stent-to-artery ratio = oversizing ratio). We set the cutoff for relevant oversizing at an oversizing ratio of $\geq 1.4$, as suggested by Zhao et al. [8]. Stents were divided into "normal-sized" and "oversized" at this cutoff point. Four animals received three stents each, one stent in the right external iliac artery and one stent in the left external iliac artery, respectively. The third stent was either placed in the right or left superficial femoral artery. In two animals, one stent was placed in the right external iliac artery and the other stent in the left external iliac artery, respectively. Six stents (4 uncoated nitinol stents and 2 TiNOX-coated stents) were overlapping a bifurcation with a significant reduction in vessel diameter distal to the bifurcation. Stent-to-artery ratio was $\geq 1.4$ distal and $<1.4$ proximal to the bifurcation. Accordingly, these stents were divided into a proximal and distal segment and each segment was analyzed separately.

Stent implantation was performed under general anesthesia, which was induced with sodium pentobarbital $10 \mathrm{mg} / \mathrm{kg}$ intravenously (IV) and maintained by halothane inhalation [10, 11]. The left carotid artery was dissected free and a 6-French vascular sheath was placed and sutured into position to perform selective angiography and stent placement into the right and left iliofemoral arteries. Stent distribution was homogenous among the animals. No stent overlapping was performed. After implantation, the animals were given a loading dose of $250 \mathrm{mg}$ of acetylsalicylic acid and then transported on the same day back to the farm, where they were housed for 5 months. After this period, pigs were brought back to the hospital and a second angiography under general anesthesia was performed in all animals. Immediately after angiography, animals were euthanized by using potassium chloride. The iliofemoral arteries were removed subsequently. The arteries with the stents were dissected and fixed in $300 \mathrm{ml}$ of buffered $4 \%$ formaldehyde. Four weeks after fixation, the stents were embedded in polymethylmethacrylate and cut with a special microtome into $800-\mu \mathrm{m}$-thin slices and polished to a thickness of $100 \mu \mathrm{m}$. All sections were prepared for light microscopy by staining with Paragon (7.3 g toluidine blue with basic fuchsin dissolved in $1,000 \mathrm{ml} 30 \%$ ethanol) under prewarming $\left(90^{\circ} \mathrm{C}\right.$ for $\left.15 \mathrm{~s}\right)$.

\section{Quantitative Angiography}

All angiograms were assessed quantitatively by a standard software program (Medis SA, Medical Imaging Systems, Leiden, The Netherlands; Fig. 1) [12]. Minimal lumen diameter (MLD) and the proximal and distal reference diameters were measured quantitatively. Late loss was calculated by subtracting MLD at follow-up from MLD at baseline. In-stent restenosis was calculated from MLD divided by the reference diameter multiplied by 100 as previously described [10-12].

\section{Histomorphometry}

After staining of the samples with Paragon, quantitative evaluation of the stented vessels was performed by using the Image-pro plus digital system (Media Cybernetics, Inc., Bethesda, MD) as previously described [11].

Histological evaluation was performed by a blinded observer $(\mathrm{NH})$ unaware of the location or coating status of the stent (Fig. 2). Within the stented iliofemoral segments, 


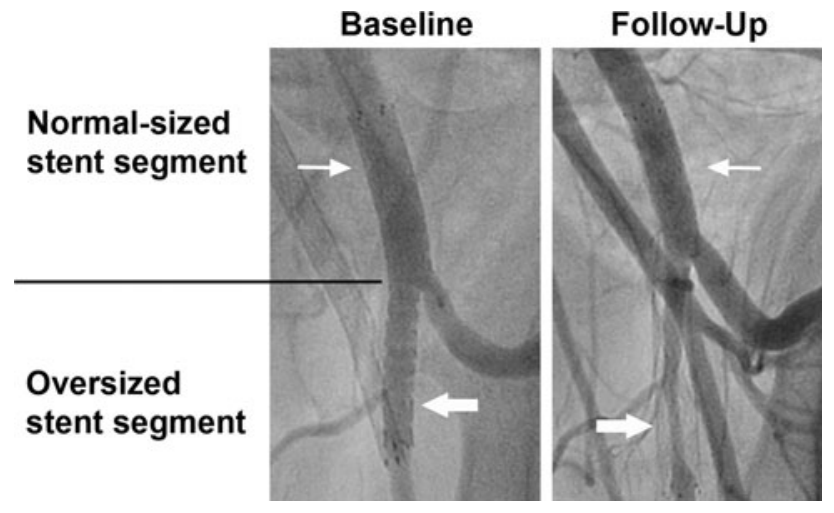

Fig. 1 Comparison of angiographic images at baseline and 5-month follow-up shows differences of in-stent restenosis at 5 months between normal-sized and oversized stent segments (lean arrows indicate normal-sized stent segments, bold arrows indicate oversized stent segments)

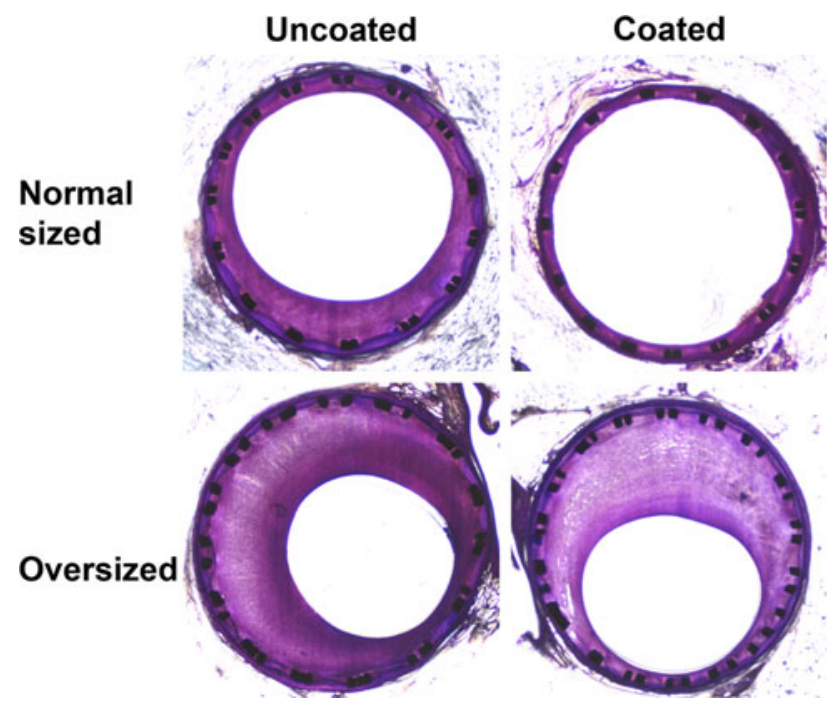

Fig. 2 Histologic micrographs (Paragon staining) at 5 months shows differences of in-stent restenosis between normal and oversized stent segments for uncoated nitinol stents (left) and titanium-nitride-oxidecoated nitinol stents (right). Magnification 10x

two sections were examined. The following parameters were determined:

1. Vessel lumen, intimal proliferation, and stent lumen (vessel lumen plus intimal proliferation). Mean and median values, as well as standard deviations were calculated for each stent section, as appropriate.

2. Intimal proliferation was calculated from the stent lumen minus the vessel lumen $\left(\mathrm{mm}^{2}\right)$ and in-stent restenosis from intimal proliferation divided by the stent lumen multiplied by 100 .

3. Reendothelialization was assessed under high-magnification light microscopy. In all sections, inflammation was visually assessed.

\section{Statistical Analysis}

Continuous variables are expressed as mean \pm standard deviation or median with ranges as appropriate. Intergroup comparisons were performed by two-tailed Student's $t$ test or Mann-Whitney test as appropriate. Within-group analyses at different time points were performed by two-tailed paired Student's $t$ test. Categorical variables are reported as counts and percentages and were compared by Fisher's exact test. Linear regressions were used for data analysis in dot-plots. N numbers represents stent segments. Statistical significance was assumed with a two-sided $P$ value $<0.05$. All data were analyzed using GraphPad Prism, version 5.

\section{Results}

All animals survived the intervention and the follow-up interval of 5 months without any clinical symptoms. One TiNOX-coated stent could not be retrieved from the animal and was therefore excluded from the study. In total, 21 stent segments were analyzed (uncoated nitinol $\mathrm{n}=14$, TiNOX-coated $n=7$ ). Of these, 12 stent segments were normal-sized and 9 were oversized. No significant difference was observed in average nominal stent diameter between the uncoated nitinol stents (range 5-6 mm; median $6 \mathrm{~mm}$ ) and TINOX-coated stents (range 5-6 mm; median $6 \mathrm{~mm} ; P=1$ ). Stent migration, stent fracture, or thrombus formation were not observed in either group. All stented artery segments were patent at 5 months (Fig. 1). The oversizing ratio ranged from 1.0 to 2.3 . The mean oversizing ratio was $1.1 \pm 0.1$ in the normal and $1.7 \pm 0.3$ in the oversized group $(P<0.001)$. Six $(29 \%)$ stent segments in the uncoated nitinol group were oversized and three (14\%) stent segments in the TiNOX group $(P=1$; Table 1). No significant difference in oversizing ratios between uncoated self-expanding stent segments and TINOX-coated stent segments existed $(1.4 \pm 0.4 \mathrm{~mm}$ vs. $1.31 \pm 0.4 \mathrm{~mm} ; P=0.58)$.

At baseline, the stent segments had a mean implantation diameter of $4.4 \pm 1.0 \mathrm{~mm}$ (range 2.6-6.2 mm; median $4.4 \mathrm{~mm}$ ), not fully expanded to their median nominal diameter of $6 \mathrm{~mm}$. Five months after implantation, all stent segments increased in diameter to $5.1 \pm 0.6 \mathrm{~mm}$ under the outward self-expanding force $(5.4 \pm 0.5 \mathrm{~mm}$ for the normal-sized, $P=0.002$ vs. baseline, and $4.8 \pm 0.6 \mathrm{~mm}$ for the oversized group, $P<0.001$ vs. baseline; Table 1). Yet, mean stent expansion was less in the normal-sized compared with the oversized group $(0.3 \pm 0.2 \mathrm{~mm}, 6 \%$ vs. $1.4 \pm 0.7 \mathrm{~mm}, 29 \% ; P=0.001)$. The changes in angiographic stent diameter for 21 individual stent segments immediately after implantation (0 day) and 5 months after implantation are shown in Fig. 3. As expected, arteries 
Table 1 Angiographic and histomorphometric data for normal-sized $(\mathrm{n}=12)$ and oversized $(\mathrm{n}=9)$ stent segments with comparison for uncoated self-expanding stent segments $(\mathrm{n}=14)$ and titanium-nitride-oxide-coated stent segments $(\mathrm{n}=7)$

\begin{tabular}{|c|c|c|c|c|c|c|}
\hline & \multicolumn{3}{|c|}{ Normal-sized } & \multicolumn{3}{|l|}{ Oversized } \\
\hline & $A L L(12)$ & UNS (8) & $\operatorname{TINOX}(4)$ & $A L L(9)$ & UNS (6) & $\operatorname{TINOX}(3)$ \\
\hline MLD BL (mm) & $4.9 \pm 0.7$ & $4.6 \pm 0.7^{\dagger}$ & $5.2 \pm 0.5$ & $3.5 \pm 0.9^{*}$ & $3.8 \pm 1.0^{* \dagger}$ & $3.0 \pm 0.3^{*}$ \\
\hline MLD FU (mm) & $4.4 \pm 0.7$ & $4.0 \pm 0.8^{\dagger}$ & $4.6 \pm 0.7$ & $2.0 \pm 1.0^{*}$ & $1.9 \pm 1.2^{*, \dagger}$ & $2.0 \pm 0.7 *$ \\
\hline Artery diameter at BL (mm) & $5.1 \pm 0.6$ & $5.0 \pm 0.7^{\dagger}$ & $5.3 \pm 0.5$ & $3.4 \pm 0.5^{*}$ & $3.3 \pm 0.5^{*, \dagger}$ & $3.6 \pm 0.5^{*}$ \\
\hline Stent diameter at FU (mm) & $5.4 \pm 0.5$ & $5.3 \pm 0.6^{\dagger}$ & $5.6 \pm 0.3$ & $4.8 \pm 0.6^{*}$ & $4.5 \pm 0.5^{*, \dagger}$ & $5.4 \pm 0.4$ \\
\hline Stent expansion at FU (mm) & $0.3 \pm 0.2^{\S}$ & $0.3 \pm 0.2^{\dagger, \S}$ & $0.3 \pm 0.3$ & $1.4 \pm 0.7^{*, \S}$ & $1.2 \pm 0.8^{*, \dagger, \S}$ & $1.7 \pm 0.1 *$ \\
\hline Oversizing ratio & $1.1 \pm 0.1$ & $1.1 \pm 0.1^{\dagger}$ & $1.0 \pm 0.1$ & $1.7 \pm 0.3^{*}$ & $1.8 \pm 0.3^{*, \dagger}$ & $1.7 \pm 0.3^{*}$ \\
\hline Angiographic late loss (mm) & $0.6 \pm 0.3$ & $0.6 \pm 0.4^{\dagger}$ & $0.6 \pm 0.2$ & $1.6 \pm 0.7^{*}$ & $1.8 \pm 0.6^{* \dagger}$ & $1.0 \pm 0.8$ \\
\hline Angiographic in-stent restenosis (\%) & $13 \pm 7$ & $13 \pm 8^{\dagger}$ & $11 \pm 4$ & $48 \pm 17 *$ & $51 \pm 18^{*, \dagger}$ & $43 \pm 15^{*}$ \\
\hline Histologic in-stent restenosis (\%) & $18 \pm 7$ & $18 \pm 8^{\dagger}$ & $18 \pm 8$ & $52 \pm 23^{*}$ & $43 \pm 22 *, \dagger$ & $66 \pm 19^{*}$ \\
\hline
\end{tabular}

Number in parenthesis indicates number of stent segments per group. $B L$ baseline, $F U 5$ months follow-up, $M L D$ minimal lumen diameter, TiNOX titanium-nitride-oxide-coated nitinol stent, UNS uncoated self-expanding nitinol stent

$* P<0.05$ normal-sized vs. oversized

${ }^{\dagger} P=$ ns UNS vs. TINOX

$\S P<0.05$ baseline vs. 5 months follow-up

before stenting were larger in the normal-sized than in the oversized segments $(5.1 \pm 0.6 \mathrm{~mm}$ vs. $3.4 \pm 0.5 \mathrm{~mm}$, $P<0.001)$. At baseline, MLD was $4.9 \pm 0.7 \mathrm{~mm}$ in the normal-sized and $3.5 \pm 0.9$ in the oversized stent group $(P=0.002)$. After 5 months, angiographic MLD was less than baseline MLD in uncoated self-expanding stents segments but also in TINOX-coated stent segments (Table 1). MLD at follow-up was $4.4 \pm 0.7 \mathrm{~mm}$ in the normal-sized and $2.0 \pm 1.0$ in the oversized stent group $(P<0.001$; Table 1). A certain amount of in-stent restenosis due to neointimal proliferation was observed in all stent segments. However, angiographic in-stent restenosis was less pronounced in the normal-sized compared with the oversized stent segments $(13 \pm 6 \%$, range $3-28$ vs. $48 \pm 17 \%$, range 25-66; $P<0.001$; Fig. 4). Accordingly, late loss was less in the normal stent group than in the oversized stent group $(0.6 \pm 0.3 \mathrm{~mm}$ vs. $1.6 \pm 0.7 \mathrm{~mm}$, $P<0.001)$. No significant differences between uncoated self-expanding stents segments and TiNOX-coated stent segments were observed $(29 \pm 23 \%$ vs. $25 \pm 19 \%$, $P=0.67$; Fig. 4). Representative angiographic and histomorphometric images of uncoated self-expanding and TiNOX-coated stents divided into normal-sized and oversized segments are shown in Figs. 1 and 2. Three stent segments (1 normal-sized uncoated, 1 oversized uncoated, and 1 normal-sized TiNOX-coated stent segment) could not be analyzed by histomorphometry due to technical problems. Histological examination of all other stent segments $(\mathrm{n}=18)$ showed fully endothelialized neointimal layers covering the inner layer of neointimal proliferation. No significant inflammation was found. Corresponding to the angiographic results, the oversized stent segments of both stent types displayed a similar degree of remarkable lumen narrowing and in-stent restenosis due to neointimal proliferation by histology $(18 \pm 7 \%$, range $8-27 \%$ for normal-sized vs. $52 \pm 23 \%$, range $23-88 \%$ for oversized,
Fig. 3 Change in individual stent diameter during 5 months postimplantation for uncoated nitinol stent segments (left panel) and titanium-nitrideoxide-coated nitinol stent segments (right panel). BL baseline, $F U$ 5-month followup. Data are given as mean $\pm \mathrm{SD}$
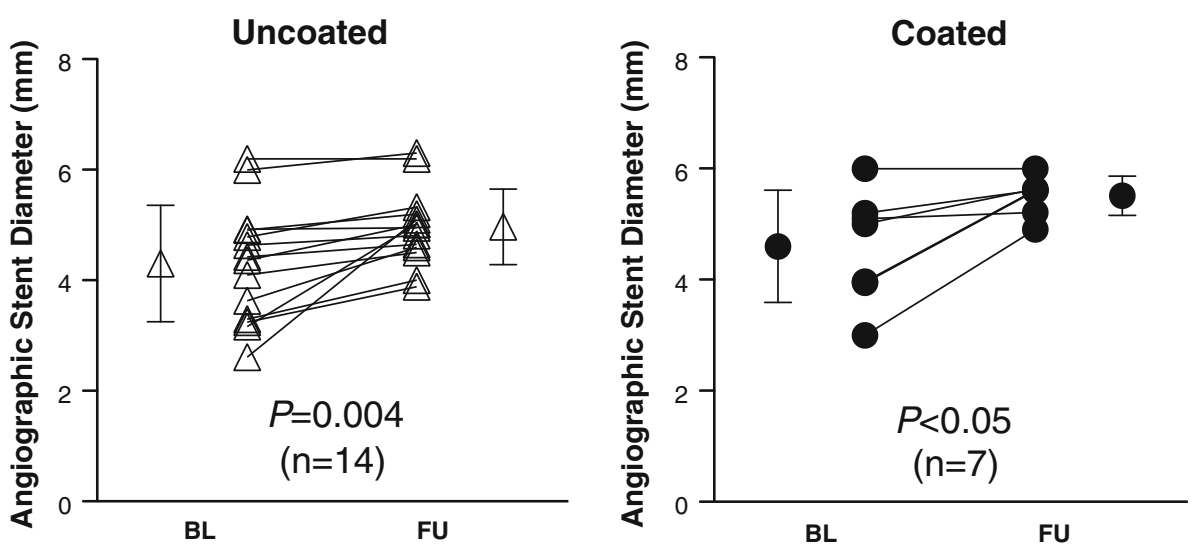
Fig. 4 Box plots displaying maximal in-stent restenosis by angiography at 5-month followup for all uncoated vs. all titanium-nitride-oxide-coated nitinol stent segments $(P=0.74$, left panel $)$ and in all normal-sized vs. all oversized stent segments $(P<0.001$, right panel). Whiskers indicate minimal and maximal values
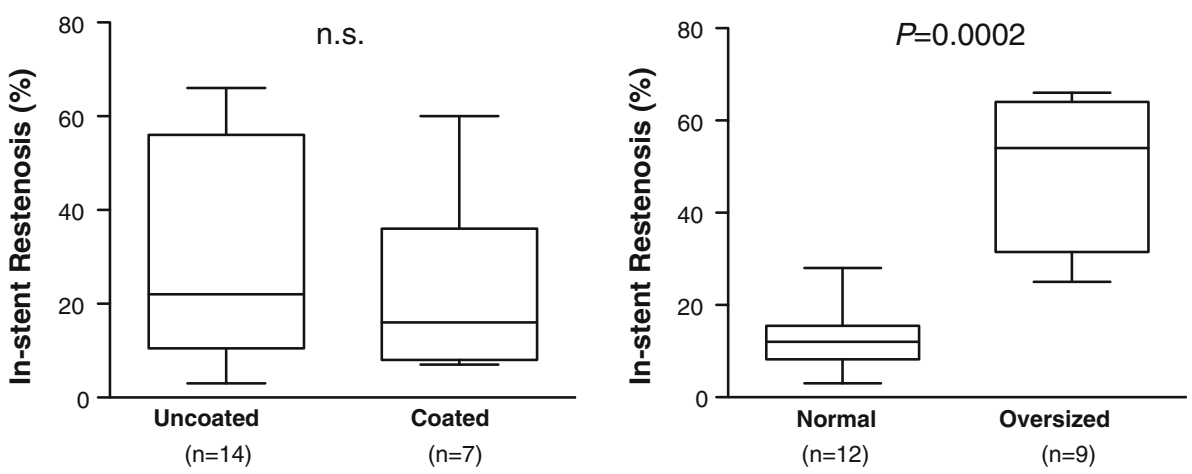

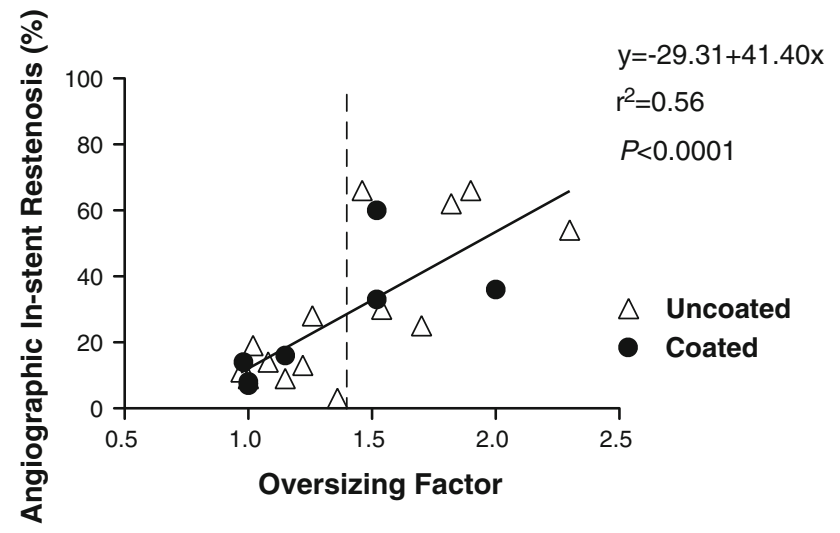

Fig. 5 A linear correlation of oversizing (nominal stent diameter-toartery ratio) and maximal in-stent restenosis obtained by quantitative angiography at 5 months postimplantation. Dotted line divides normal-sized from oversized stent segments (nominal stent diameter-to-artery ratio $=$ oversizing factor of 1.4 )

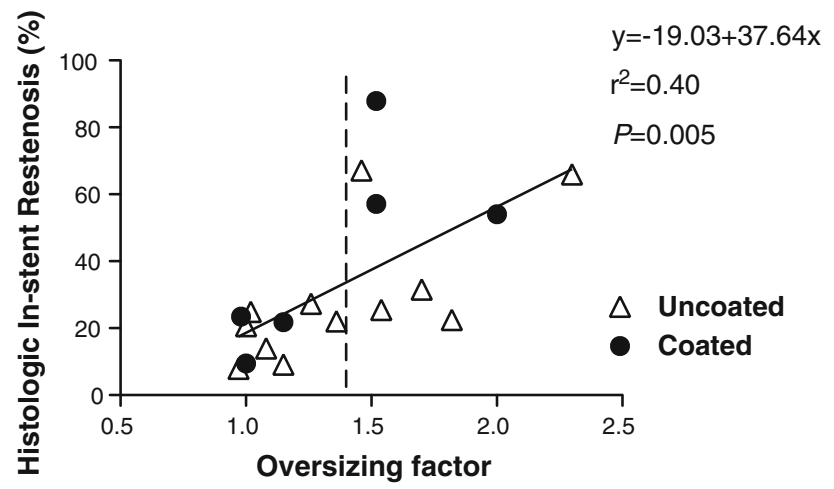

Fig. 6 A linear correlation of oversizing (nominal stent diameter-toartery ratio) and maximal in-stent luminal area restenosis obtained by histomorphometry at 5 months postimplantation. Dotted line divides normal-sized from oversized stent segments (nominal stent diameterto-artery ratio $=$ oversizing factor of 1.4 )

$P<0.001$; Fig. 2). As for the angiographic results, no significant differences between uncoated self-expanding stents segments and TiNOX-coated stents segments were observed with regard to histomorphometric in-stent restenosis $(28 \pm 19 \%$ vs. $42 \pm 29 \%, P=0.23)$. A dot-plot of angiographic and histomorphometric in-stent restenosis at 5 months against the oversizing ratio among all analyzed samples are shown in Figs. 5 and 6. Linear regression analysis showed a positive linear correlation between oversizing ratio and angiographic $\left(R^{2}=0.56, P<0.0001\right)$ as well as histomorphometric $\left(R^{2}=0.4, P=0.005\right)$ instent restenosis (Figs. 5, 6). Separate analysis for uncoated self-expanding stent segments and TiNOX-coated stents segments yielded similar results (Table 1).

\section{Discussion}

The data of the present study show that TiNOX-coated selfexpanding nitinol stents are as safe and effective as uncoated self-expanding nitinol stents in the porcine iliofemoral artery with an excellent outcome after 5 months in both groups. The implantation procedure was successful in all animals, late follow-up was uneventful, and animals showed no limitations in physical activity and range of motion during the observed postinterventional period. In the present study, histomorphometry importantly showed patency in all samples with open stents in all examined segments. However, neointimal proliferation of more than $25 \%$ was observed in areas with oversized stent segments (oversizing ratio $\geq 1.4$ ). During the follow-up period of 5 months, stents increased in diameter by $6 \%$ in the normal-sized and $29 \%$ in the oversized group, almost reaching their nominal diameter under the outward expanding force of the selfexpanding stent. Mean stent expansion after 5 months was significantly greater for oversized stents. This finding may be explained by the fact that oversized stents were mostly deployed in smaller arteries featuring greater counter-force at implantation [13], but storing higher strain energy in the stent that leads to pronounced stent expansion over time [8]. Gradual stent expansion for self-expanding stents is known to be different from balloon-expanding stents. In the latter, the vessel wall is immediately stretched to its definite diameter causing instantaneous injury to the artery wall.

Our data support a critical cutoff for oversizing selfexpanding stents at a ratio of 1.4 as recently proposed by 
Zhao et al. [8]. Moreover, our results also suggest that this cutoff point may be valid not only for uncoated selfexpanding nitinol stents but also for self-expanding TiNOX-coated stents.

Angiographic and histomorphometric follow-up data observed in our study demonstrate that oversizing linearly and positively correlates with neointimal proliferation and in-stent restenosis. A fourfold increase of in-stent restenosis was observed when the oversizing ratio increased by $65 \%$. This phenomenon is unique for self-expanding stents and has previously been described in coronary and carotid artery stenting $[8,14-16]$. Thus, the benefits of oversizing self-expanding stents to ensure optimal wall apposition and to prevent stent migration may be compromised by neointimal proliferation and significant in-stent restenosis. To our knowledge, Zhao et al. constitute the first and only group to demonstrate this phenomenon for uncoated selfexpanding stents in porcine iliofemoral arteries [8]. Yet, they pose the question of whether coated self-expanding stents may reduce neointima formation in oversized stents. To address this important issue, we compared uncoated self-expanding nitinol stents to TiNOX-coated selfexpanding stents. TiNOX-coated stents are biologically inert compared with the nickel component of nitinol stents. As previously shown, neointimal hyperplasia was reduced up to $50 \%$ for TiNOX-coated stents compared with stainless steel stents in porcine coronary arteries at 6 weeks of follow-up [10]. In our study, however, adequate sizing was the most important determinant of in-stent restenosis. For stents with an oversizing ratio $<1.4$, in-stent restenosis due to neointimal proliferation was minimal, and no differences were observed between uncoated self-expanding nitinol stents and TiNOX-coated stents. For an oversizing ratio $\geq 1.4$ in-stent restenosis occurred to a similar extent in uncoated self-expanding nitinol stent but also in TiNOXcoated stents (Fig. 4).

As previously shown, continuing expansion of oversized self-expanding stents may traumatize the arterial wall causing injury of the endothelium that is different than observed with balloon-expanding stent implantation [17]. This leads to an early inflammatory reaction with gradual infiltration of neutrophils and monocytes. Interactions of inflammatory cells and stent material lead to a healing process in which a fibrous capsule and neointimal layer is formed [8]. Our study indicates that the antiproliferative feature of TiNOX may not significantly alter this healing process and restenosis in the iliofemoral artery bed associated with oversizing. Because our data were obtained in a small sample size, they are preliminary and rather observational. The antiproliferative properties of TiNOX-coated stents need further investigation in larger studies [10]. Several studies have demonstrated significant improvements with self-expanding nitinol stents compared with stainless steel stents for peripheral stenting $[18,19]$. Thus, currently self-expanding nitinol stents are most frequently used for treating patients with peripheral vascular disease. However, significant restenosis and low patency rates with peripheral self-expanding nitinol stents, particularly in superficial femoral artery lesions, still pose a common clinical problem [20-22]. Initial results for drug-eluting self-expanding peripheral stents were promising [7, 23, 24]. Unfortunately, subsequent studies with sirolimus- and everolimus-eluting self-expanding nitinol stents failed to demonstrate improved mid-term patency compared with bare-metal stents $[24,25]$. These findings were certainly a surprise, because drug-eluting stents constituted a major step forward in the treatment of coronary artery disease, namely a 50-70\% reduction in restenosis compared with bare-metal stents. Several mechanisms may be responsible for this differential efficacy of drug-eluting stents in coronary versus peripheral arteries.

First, peripheral vessels display an entirely different remodeling behavior of the vessel wall, following the placement of self-expanding stents compared with balloonexpanding stents implanted in coronary arteries [8]. This may be partly explained by a different anatomy, e.g., the higher elasticity of the iliofemoral artery compared with the coronary artery, resulting in a lower degree of instantaneous expansion. However, higher strain energy is stored in the self-expanding stent, which may lead to enhanced time-dependent expansion, vessel injury, and neointimal hyperplasia [8, 13]. Moreover, femoral arterial segments have to face stronger external physical forces, such as compression, elongation, and torsion that promote restenosis [26]. Differences in blood flow and shear stress in different vascular regions also have to be considered. These biologic and mechanical differences may explain why the potential benefit observed with TiNOX stents compared with bare-metal stents in the coronary artery bed could not be reproduced in the iliofemoral arteries in our study $[10,11]$. Of note, clinical investigations have demonstrated that the concept of TiNOX coating might be superior to stainless steel stenting [27] but inferior to newer generation drug-eluting stents with regard to the prevention of restenosis in patients with coronary artery disease [28].

Finally, it is noteworthy that recent data of a new paclitaxel-eluting self-expanding nitinol stent were promising with respect to patency rates; however, only long-term data will show whether the superiority in terms of efficacy can be sustained [29].

In summary, as recently proposed by Zhao et al. [8] for uncoated nitinol stents and now demonstrated by our data for TiNOX-coated self-expanding nitinol stents, exact sizing of self-expanding stents without exceeding an oversizing ratio of 1.4 seems to be crucial for long-term patency in peripheral artery stenting. 


\section{Limitations}

First, the sample size of 15 stents is small. Thus, the current study may be underpowered to show statistical differences in restenosis between both stent types. However, certain restrictions of the local ethical committee regarding the use of pigs for the current study did not allow a higher number of study animals. Second, this study was performed in healthy animals with nonatherosclerotic arteries. Although the porcine vasculature is very similar to that of humans [30], our data may not be fully transferred to humans, particularly not to patients with peripheral artery disease with different lesion lengths and diameters that may react variable to TiNOX stenting.

Third, baseline reference vessel diameters were different between oversizing and normal-sizing stent groups, which may have impacted in part on the differences in restenosis that we observed in addition to the effect of stent oversizing, potentially due to differences between these vessel segments per se. However, our data indicate that relevant restenosis also was observed in larger vessel segments, if stent-to-artery ratio exceeded 1.3. Fourth, the stents of the manufacturers had differences in length and diameter, which was unavoidable, because stents were implanted as distributed by the vendors at the time of the study. Fifth, only a single intravenous dose of $250 \mathrm{mg}$ of acetylsalicylic acid was administered periprocedural, in full accordance with previously published studies by our group [11], but in contrast to recent porcine peripheral stent studies implementing dual antiplatelet therapy for 1 month [31]. Although this may increase the propensity for acute and early stent thrombosis, we did not observe any total occlusions at follow-up, and no data are available that would submit a correlation of the length of antiplatelet therapy and late lumen loss in the absence of stent thrombosis.

\section{Conclusions}

In this experimental model of peripheral endovascular intervention, we were able to demonstrate that selfexpanding TiNOX-coated stents are as safe and effective as uncoated self-expanding nitinol stents. Both stent types were constrained by their target artery at implantation and expanded over time to near their nominal diameter within 5 months. Severe oversizing determined as an oversizing ratio $\geq 1.4$ resulted in significant neointimal proliferation and in-stent restenosis, which may not be reduced by TiNOX-coating.

Acknowledgments The study was supported by an unrestricted grant of Hexacath SA, Paris, France. The authors thank O. Beslac for expert technical assistance. This work is dedicated to our mentor, Otto M. Hess, who is deeply missed.

Conflicts of interest The authors do not have any conflicts of interest to report.

\section{References}

1. Schurmann K, Mahnken A, Meyer J et al (2002) Long-term results 10 years after iliac arterial stent placement. Radiology 224:731-738

2. Schillinger M, Sabeti S, Loewe C et al (2006) Balloon angioplasty versus implantation of nitinol stents in the superficial femoral artery. N Engl J Med 354:1879-1888

3. Laird JR, Katzen BT, Scheinert D et al (2010) Nitinol stent implantation versus balloon angioplasty for lesions in the superficial femoral artery and proximal popliteal artery: twelvemonth results from the RESILIENT randomized trial. Circ Cardiovasc Interv 3:267-276

4. Karthik S, Tuite DJ, Nicholson AA et al (2007) Cryoplasty for arterial restenosis. Eur J Vasc Endovasc Surg 33:40-43

5. Vorwerk D, Redha F, Neuerburg J et al (1994) Neointima formation following arterial placement of self-expanding stents of different radial force: experimental results. Cardiovasc Intervent Radiol 17:27-32

6. Schurmann K, Vorwerk D, Kulisch A et al (1996) Neointimal hyperplasia in low-profile nitinol stents, palmaz stents, and wallstents: a comparative experimental study. Cardiovasc Intervent Radiol 19:248-254

7. Zeller T (2007) Current state of endovascular treatment of femoro-popliteal artery disease. Vasc Med 12:223-234

8. Zhao HQ, Nikanorov A, Virmani R et al (2009) Late stent expansion and neointimal proliferation of oversized nitinol stents in peripheral arteries. Cardiovasc Intervent Radiol 32:720-726

9. Stoeckel D, Pelton A, Duerig T (2004) Self-expanding nitinol stents: material and design considerations. Eur Radiol 14: 292-301

10. Windecker S, Mayer I, De Pasquale G et al (2001) Stent coating with titanium-nitride-oxide for reduction of neointimal hyperplasia. Circulation 104:928-933

11. Flueckiger A, Strahm Y, Billinger M et al (2009) Intimal proliferation and restenosis in paclitaxel-eluting stents with aminoparylene as carrier substance in swines. J Invasive Cardiol $21: 128-132$

12. Billinger M, Buddeberg F, Hubbell JA et al (2006) Polymer stent coating for prevention of neointimal hyperplasia. J Invasive Cardiol 18:423-426 discussion 427

13. Liu Y, Dang C, Garcia M et al (2007) Surrounding tissues affect the passive mechanics of the vessel wall: theory and experiment. Am J Physiol Heart Circ Physiol 293:H3290-H3300

14. Schwartz RS, Huber KC, Murphy JG et al (1992) Restenosis and the proportional neointimal response to coronary artery injury: results in a porcine model. J Am Coll Cardiol 19:267-274

15. Han RO, Schwartz RS, Kobayashi Y et al (2001) Comparison of self-expanding and balloon-expandable stents for the reduction of restenosis. Am J Cardiol 88:253-259

16. Clark DJ, Lessio S, O’Donoghue M et al (2006) Mechanisms and predictors of carotid artery stent restenosis: a serial intravascular ultrasound study. J Am Coll Cardiol 47:2390-2396

17. Carter AJ, Laird JR, Farb A et al (1994) Morphologic characteristics of lesion formation and time course of smooth muscle cell proliferation in a porcine proliferative restenosis model. J Am Coll Cardiol 24:1398-1405 
18. Sabeti S, Schillinger M, Amighi J et al (2004) Primary patency of femoropopliteal arteries treated with nitinol versus stainless steel self-expanding stents: propensity score-adjusted analysis. Radiology 232:516-521

19. Sabeti S, Mlekusch W, Amighi J et al (2005) Primary patency of long-segment self-expanding nitinol stents in the femoropopliteal arteries. J Endovasc Ther 12:6-12

20. Ansel GM, Lumsden AB (2009) Evolving modalities for femoropopliteal interventions. J Endovasc Ther 16:II82-II97

21. Krankenberg H, Schluter M, Steinkamp HJ et al (2007) Nitinol stent implantation versus percutaneous transluminal angioplasty in superficial femoral artery lesions up to $10 \mathrm{~cm}$ in length: the femoral artery stenting trial (FAST). Circulation 116:285-292

22. Kasapis C, Henke PK, Chetcuti SJ et al (2009) Routine stent implantation vs. percutaneous transluminal angioplasty in femoropopliteal artery disease: a meta-analysis of randomized controlled trials. Eur Heart J 30:44-55

23. Duda SH, Bosiers M, Pusich B et al (2002) Endovascular treatment of peripheral artery disease with expanded PTFE-covered nitinol stents: interim analysis from a prospective controlled study. Cardiovasc Intervent Radiol 25:413-418

24. Duda SH, Bosiers M, Lammer J et al (2005) Sirolimus-eluting versus bare nitinol stent for obstructive superficial femoral artery disease: the SIROCCO II trial. J Vasc Interv Radiol 16:331-338
25. Lammer J, Bosiers M, Zeller T et al (2011) First clinical trial of nitinol self-expanding everolimus-eluting stent implantation for peripheral arterial occlusive disease. J Vasc Surg 54:394-401

26. Tang GL, Morasch MD (2007) Role of stents, drug-eluting stents, and stent-grafts in treatment of infrainguinal arterial disease. Semin Vasc Surg 20:37-41

27. Windecker S, Simon R, Lins M et al (2005) Randomized comparison of a titanium-nitride-oxide-coated stent with a stainless steel stent for coronary revascularization: the TiNOX trial. Circulation 111:2617-2622

28. Pilgrim T, Raber L, Limacher A et al (2011) Comparison of titanium-nitride-oxide-coated stents with zotarolimus-eluting stents for coronary revascularization a randomized controlled trial. JACC Cardiovasc Interv 4:672-682

29. Minar E (2011) Drug-eluting stents above the knee. J Cardiovasc Surg (Torino) 52:225-229

30. Traupe T, Stettler CD, Li H et al (2007) Distinct roles of estrogen receptors alpha and beta mediating acute vasodilation of epicardial coronary arteries. Hypertension 49:1364-1370

31. Dake MD, Van Alstine WG, Zhou Q et al (2011) Polymer-free paclitaxel-coated Zilver PTX Stents-evaluation of pharmacokinetics and comparative safety in porcine arteries. J Vasc Interv Radiol 22:603-610 